\title{
T-box 2 expression predicts poor prognosis in gastric cancer
}

\author{
HAIHUA YU*, BO LIU*, AIWU LIU, KAI LI and HONGPENG ZHAO \\ Department of Gastrointestinal Surgery, Shandong Provincial Qianfoshan Hospital, Jinan, Shandong 250014, P.R. China
}

Received September 8, 2014; Accepted June 3, 2015

DOI: $10.3892 / 01.2015 .3428$

\begin{abstract}
T-Box 2 (TBX2) plays a critical role in embryonic development. Previously, TBX2 has been suggested to be involved in malignancies. However, the role of TBX2 in gastric cancer (GC) remains unclear. In the present study, TBX2 expression was found to be evidently upregulated in GC compared with the expression in the corresponding adjacent non-cancerous tissues at the mRNA and protein levels. Using immunohistochemical analysis on the tissue samples obtained from 266 patients with GC, TBX2 expression was found to be significantly associated with the clinical stage and incidence of vascular invasion and metastasis. Patients with GC that expressed TBX2 demonstrated a shorter overall survival time compared with the patients without TBX2 expression. Multivariate analysis revealed that TBX2 expression was an independent prognostic factor for overall survival (hazard ratio, 3.930; 95\% confidence interval, 2.041-7.917; $\mathrm{P}=0.009$ ). These results indicated that TBX2 is a prognostic marker for patients with GC.
\end{abstract}

\section{Introduction}

Gastric cancer (GC) is an aggressively invasive tumor, and one of the most common lethal cancers worldwide (1-2). Surgical resection may successfully treat GC in the early stage of disease (3). However, a considerable number of patients with GC are diagnosed at an advanced stage of disease, resulting in a poor prognosis (4). Thus, exploring the potential novel markers of GC is critical to make an early diagnosis, guide the treatment regimens and predict the prognosis of patients with GC.

T-box 2 (TBX2) is a member of the T-box family of transcription factors, which are involved in embryonic development (5). In addition to playing a role in development, it has been suggested that TBX2 is involved in cell cycle regulation

Correspondence to: Dr Hongpeng Zhao, Department of Gastrointestinal Surgery, Shandong Provincial Qianfoshan Hospital, 16766 Jingshi Avenue, Jinan, Shandong 250014, P.R. China

E-mail: zhao_hongpeng@126.com

*Contributed equally

Key words: gastric cancer, T-box 2, prognosis, biomarker and cancer (6). Deregulated TBX2 gene expression is found in breast cancer (7), melanoma (8) and pancreatic cancer (9). However, the expression and role of TBX2 in GC have yet to be fully elucidated. In the present study, the expression of TBX2 in GC was investigated using reverse transcription-quantitative polymerase chain reaction (RT-qPCR), western blotting and immunohistochemistry. The correlation between the expression of TBX2 and the clinicopathological features of patients with GC was then analyzed.

\section{Patients and methods}

Patients. In total, 25 fresh samples of GC and paired adjacent non-cancerous tissues were obtained between May 1, 2013 and August 1, 2013 at Shandong Provincial Qianfoshan Hospital (Jinan, China), and used for RT-qPCR and western blot analysis to determine the TBX2 expression levels. In addition, tissue samples obtained from 266 patients that underwent surgery for the treatment of GC between January 1, 2004 and December 1, 2008 at Shandong Provincial Qianfoshan Hospital were used for immunohistochemical analysis. The group included 371 men and 245 women, with a mean age of $62.6 \pm 17.4$ years. The present study was approved by the Ethics Committee of Shandong Provincial Qianfoshan Hospital and was performed in accordance with the 2000 Declaration of Helsinki (10). Written informed consent was obtained from all patients prior to surgery.

Follow-up. All tissue samples were used for immunohistochemical analysis. All patients underwent gastrectomy, and were monitored by chest, abdominal and pelvic computed tomography (CT) and blood testing at three-month intervals and yearly gastroscopy. The overall survival time was defined as the time between surgery and clinically or radiologically confirmed recurrence or metastasis, or mortality.

$R T-q P C R$. Total RNA was extracted using TRIzol reagent (Invitrogen, Carlsbad, CA, USA) and reverse transcribed into cDNA using the Reverse Transcription kit (Invitrogen). The cDNA was then amplified by RT-qPCR using the SYBR Green PCR kit (Invitrogen). The primers used for PCR were as follows: TBX2 forward, 5'-TGCGGAGAACTCCAGGTT-3' and reverse, 5'-AGCTGGTGAGTGGAGCCA-3'; and GAPDH forward, 5'-ACAGTCAGCCGCATCTTCTTTTG-3' and reverse, 5'-CCCACTTGATTTTGGAGGGATCT-3'. The data were analyzed using the $\Delta \Delta \mathrm{Ct}$ method and the expression of TBX2 was normalized against the GAPDH transcripts. 
Western blot analysis. Fresh tumor sections obtained from 25 patients recently diagnosed with GC and the corresponding adjacent non-cancerous tissues were lysed in cell lysis buffer (Sigma-Aldrich, St. Louis, MO, USA). The samples were homogenized and then maintained at $4^{\circ} \mathrm{C}$ for $30 \mathrm{~min}$. Cell extracts were centrifuged at $12,000 \mathrm{x} \mathrm{g}$ for $30 \mathrm{~min}$ at $4^{\circ} \mathrm{C}$ and the protein concentration was determined using a bicinchoninic acid kit (Pierce Biotechnology, Inc., Rockford, IL, USA). Total protein $(50 \mu \mathrm{g})$ from each sample was heated at $95^{\circ} \mathrm{C}$ for $5 \mathrm{~min}$ subsequent to mixing with an equal volume of $2 X$ SDS loading buffer. The samples were separated on $12.5 \%$ SDS-PAGE gels and transferred to polyvinylidene difluoride membranes (EMD Millipore, Billerica, MA, USA). The membrane was blocked in Tris-buffered saline with Tween 20 (TBS-T) containing 5\% skim milk at room temperature for $2 \mathrm{~h}$. The membranes were incubated with TBX2 antibody (dilution, 1:2,000) in TBS-T overnight at $4^{\circ} \mathrm{C}$. Subsequent to washing with TBS-T, the membrane was incubated in 5\% skim milk in TBS-T buffer containing a secondary antibody (dilution, 1:5,000) for $60 \mathrm{~min}$ at room temperature. Proteins of interest were detected using a Chemiluminescence Reagent Plus kit (Perkin Elmer, Beaconsfield, Buckinghamshire, UK). For normalization of protein loading, the monoclonal antibody against GAPDH (dilution, $1: 5,000)$ was used.

Immunohistochemistry. Immunohistochemical analysis was performed on the formalin-fixed paraffin-embedded sections of GC tumor tissues and the corresponding adjacent benign tissue specimens. The slides were microwaved in citrate buffer antigen retrieval solution (Vector Laboratories, Inc., Burlingame, CA, USA) and washed with phosphate-buffered saline (PBS) for $5 \mathrm{~min}$. The slides were then blocked with 10\% normal goat serum in PBS for 30 min and incubated overnight in the presence of rabbit polyclonal IgG anti-TBX2 antibody (dilution, 1:50; cat no. sc-48780; Santa Cruz Biotechnology, Inc., Dallas, TX, USA). The slides were then washed three times using PBS and incubated with goat anti-rabbit secondary antibody (dilution, 1:500) for $30 \mathrm{~min}$. The slides were counterstained with light green (Beijing Bitab Biotechnology Co., Ltd., Beijing, China) and evaluated semi-quantitatively based on the intensity and percentage of staining. The TBX2 staining was classified as strong (3+), moderate (2+), weak (1+) or absent (-) compared with the adjacent non-tumor tissues. Scores of 0 or $1+$ were considered to indicate a lack of TBX2 expression, and scores of $2+$ or $3+$ were considered to indicate the presence of TBX2 expression. No staining was observed using the isotype control immunoglobulin $\mathrm{G}$, indicating stain specificity.

Statistical analysis. The $\chi^{2}$ and Fisher's exact tests were used to compare categorical variables. Kaplan-Meier plot and log-rank tests were used for survival analysis. Univariate and multivariate analyses were based on the Cox proportional hazards regression model. Statistical analyses were performed using SPSS 15.0 software (SPSS, Inc., Chicago, IL, USA). P $<0.05$ was considered to indicate a statistically significant difference.

\section{Results}

Increased TBX2 mRNA and protein expression in human GC tissues. The mRNA level of TBX2 was determined by

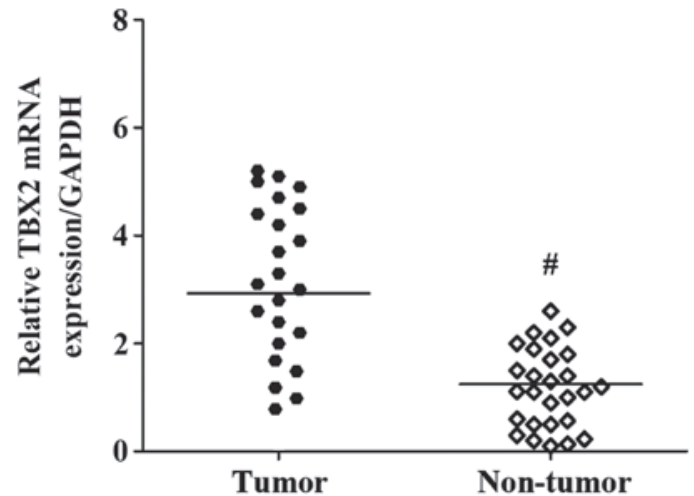

Figure 1. Increased mRNA expression of TBX2 in gastric cancer tissues determined by reverse transcription-quantitative polymerase chain reaction and normalized against GAPDH expression $\left(n=25\right.$; $\left.{ }^{\#} \mathrm{P}<0.001\right)$. Horizontal lines represent the mean. TBX2, T-box 2 .

RT-qPCR in 25 fresh GC specimens and paired corresponding adjacent non-cancerous gastric tissues. The TBX2 gene expression level was significantly increased in the cancerous tissues compared with the adjacent non-cancerous tissues ( $\mathrm{P}<0.001$; Fig. 1).

Furthermore, western blot analysis was performed on the aforementioned specimens. Consistently, the results revealed that the expression of the TBX2 protein was significantly increased in the GC tissues compared with the matched adjacent non-cancerous tissues, as semi-quantified by densitometry ( $\mathrm{P}<0.001$; Fig. 2).

Immunohistochemical analysis of TBX2 expression and the association with clinicopathological parameters. To investigate the clinicopathological significance of TBX2 expression, immunohistochemical analysis was performed using 266 paraffin-embedded GC tissue blocks. The results of the immunohistochemical analysis were graded for the expression of TBX2 as without TBX2 expression or positive for TBX2 expression. As revealed in Fig. 3, the expression rate of TBX2 in GC samples was 66.2\% (176/266). By contrast, only $8.27 \%(22 / 266)$ of paired adjacent non-cancerous tissues exhibited TBX2 expression. The association between the expression of TBX2 and various clinicopathological parameters is listed in Table I. The results revealed that the increased expression of TBX2 was significantly associated with the clinical stage of disease $(\mathrm{P}=0.022)$, incidence of vascular invasion $(\mathrm{P}=0.031)$ and presence of metastasis $(\mathrm{P}=0.005)$.

Association between TBX2 expression and prognosis, as determined using the Cox proportional hazards model. The prognostic effect of TBX2 expression on the survival of patients with GC was analyzed. The results revealed that the overall survival time of the patients with GC that demonstrated TBX2 expression was shorter compared with the survival time of patients without TBX2 expression ( $\mathrm{P}=0.007$; Fig. 4).

Univariate analysis revealed that the overall survival time of patients was significantly associated with the tumor stage and presence of vascular invasion, metastasis and expression of TBX2 (Table II). In addition, the association between the presence of TBX2 expression and survival time was found to 
Table I. Association between TBX2 expression and clinicopathological parameters in 266 patients with gastric cancer.

TBX2 expression

Parameters Total, $\mathrm{n}$ Present, $\mathrm{n}$ Absent, $\mathrm{n}$ P-value

\begin{tabular}{lrrr}
\hline Total & 266 & 176 & 90 \\
Age & & & \\
$<48$ & 100 & 62 & 38 \\
$\geq 48$ & 166 & 114 & 52
\end{tabular}

Gender

$\begin{array}{lrrr}\text { Male } & 161 & 102 & 59 \\ \text { Female } & 105 & 74 & 31\end{array}$

Clinical stage

I-II

III-IV

68

198

32

144

T-stage

$\mathrm{T} 1-\mathrm{T} 2$

T3-T4

90

176

56

120

NS

34

N-stage

N0

N1-N3

78

188

50

126

NS

28

62

Vascular invasion

No

$185 \quad 148$

37

Yes

$81 \quad 28$

53

Metastasis

$\begin{array}{lrrr}\text { No } & 200 & 121 & 79 \\ \text { Yes } & 66 & 55 & 11\end{array}$

NS, not significant; TBX2, T-box 2; T-stage, tumor stage; N-stage, stage of lymph node involvement.

A

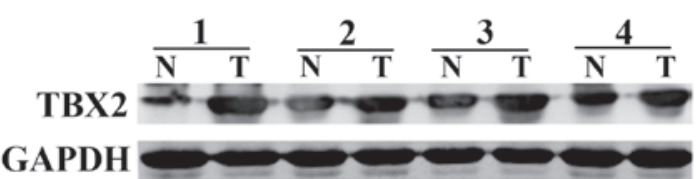

B

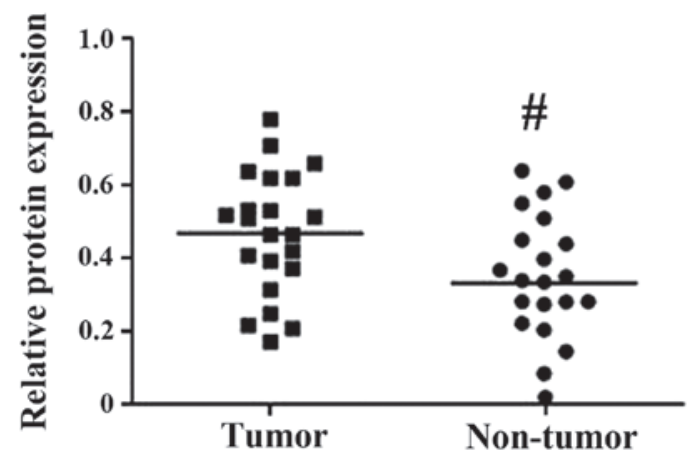

Figure 2. Increased expression of the TBX2 protein in gastric cancer tumor tissue specimens, as assessed by western blotting. (A) Representative result of TBX2 protein expression in four paired gastric tumor and adjacent non-tumor tissue samples. (B) Relative expression levels of the TBX2 protein in gastric cancer tissues and non-cancerous tissues, normalized against GAPDH expression $\left(\mathrm{n}=25 ;{ }^{\prime \prime} \mathrm{P}<0.001\right)$. Lines represent the median values. $\mathrm{N}$, non-cancerous gastric mucosa; T, gastric cancer tissues; TBX2, T-box 2 .
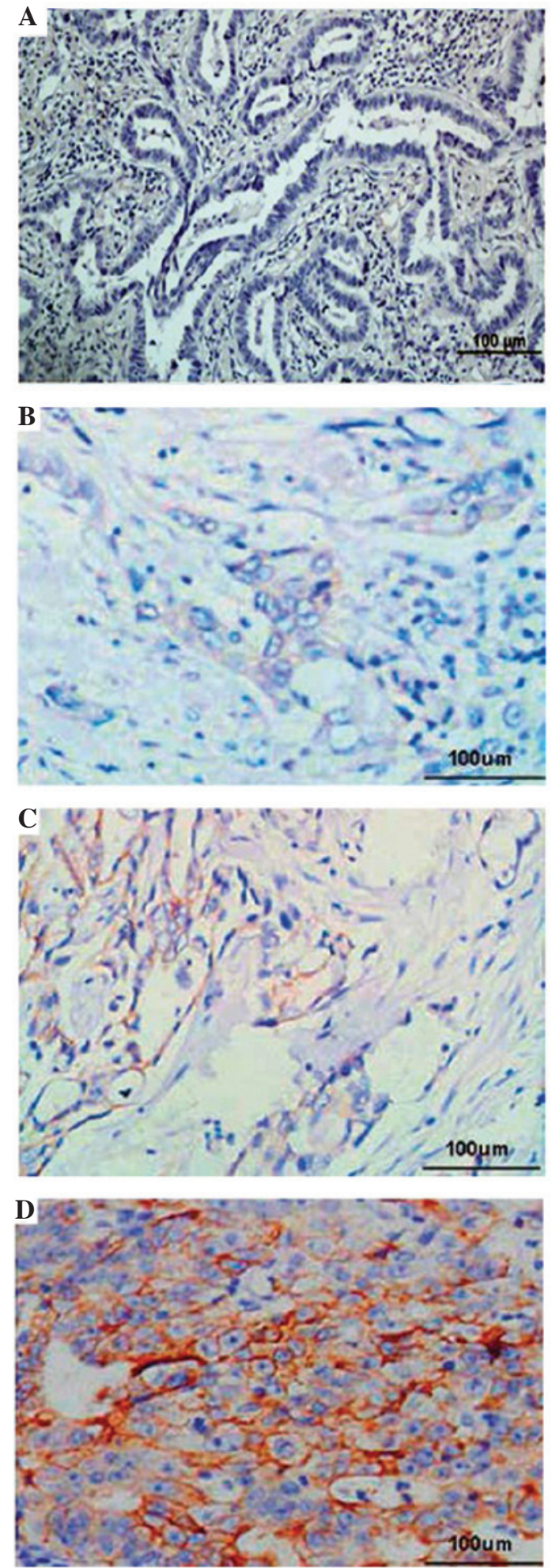

Figure 3. Representative samples of the immunohistochemical scoring of gastric cancer tissues. TBX2 expression was assessed by immunohistochemistry in gastric cancer tissue specimens by detecting the protein expression of TBX2 and scoring the immunohistochemical results using the 0 to $3+$ scale. Scores of (A) 0 or (B) 1+ were considered to not express TBX2, but (C) $2+$ and (D) 3+ were considered to be positive for TBX2 expression. Scale bar, $100 \mu \mathrm{m}$. TBX2, T-box 2 .

remain significant subsequent to controlling other prognostic factors in multivariate analysis [hazard ratio (HR), 3.930; $95 \%$ confidence interval (CI), 2.041-7.917; $\mathrm{P}=0.009$; Table III]. 
Table II. Univariate analysis of the prognosis in 266 patients with gastric cancer.

\begin{tabular}{lcc}
\hline Variables & HR $(95 \% \mathrm{CI})$ & P-value \\
\hline $\begin{array}{l}\text { Depth of invasion } \\
\text { T1+T2 vs. T3+T4 }\end{array}$ & $0.984(0.301-1.996)$ & 0.086 \\
$\begin{array}{l}\text { Nodal status } \\
\text { N0 vs. N1+N2+N3 }\end{array}$ & $0.712(0.165-1.523)$ & 0.125 \\
$\begin{array}{l}\text { Metastasis } \\
\text { Absent vs. present }\end{array}$ & $5.292(2.009-12.327)$ & $<0.001$ \\
$\begin{array}{l}\text { Stage } \\
\text { I+II vs. III+IV }\end{array}$ & $2.573(1.410-5.694)$ & 0.019 \\
$\begin{array}{l}\text { Vascular invasion } \\
\text { Absent vs. present }\end{array}$ & $3.112(1.271-6.044)$ & $<0.001$ \\
$\begin{array}{l}\text { TBX2 expression } \\
\text { Present vs. absent }\end{array}$ & $3.018(1.979-7.208)$ & 0.002 \\
\hline
\end{tabular}

HR, hazard ratio; CI, confidence interval; TBX2, T-box 2.

Table III. Multivariate analysis of the prognosis in patients with gastric cancer.

\begin{tabular}{lcc}
\hline Variables & HR $(95 \% \mathrm{CI})$ & P-value \\
\hline $\begin{array}{l}\text { Depth of invasion } \\
\text { T1+T2 vs. T3+T4 }\end{array}$ & $0.921(0.132-2.413)$ & 0.093 \\
$\begin{array}{l}\text { Nodal status } \\
\text { N0 vs. N1+N2+N3 }\end{array}$ & $1.099(0.264-3.073)$ & 0.098 \\
$\begin{array}{l}\text { Metastasis } \\
\text { Absent vs. present }\end{array}$ & $9.320(3.117-19.344)$ & $<0.001$ \\
$\begin{array}{l}\text { Stage } \\
\text { I+II vs. III+IV }\end{array}$ & $0.902(0.305-2.098)$ & 0.076 \\
$\begin{array}{l}\text { Vascular invasion } \\
\text { Absent vs. present }\end{array}$ & $1.874(1.003-4.615)$ & 0.020 \\
$\begin{array}{l}\text { TBX2 expression } \\
\text { Present vs. absent }\end{array}$ & $3.930(2.041-7.917)$ & 0.009 \\
\hline
\end{tabular}

HR, hazard ratio; CI, confidence interval; TBX2, T-box 2.

Multivariate analysis also revealed metastasis and vascular invasion to be independent prognostic factors.

\section{Discussion}

Evolutionarily conserved genes perform critical and well-established roles in embryonic development. T-box factors have gained increasing prominence in the field of cancer biology, as a wide range of cancers exhibit deregulated expression of T-box factors that possess tumor suppressor or tumor promoter functions. TBX2 is one of the best characterized members of the T-box family of transcription factors and contributes directly to tumor progression, and particularly to

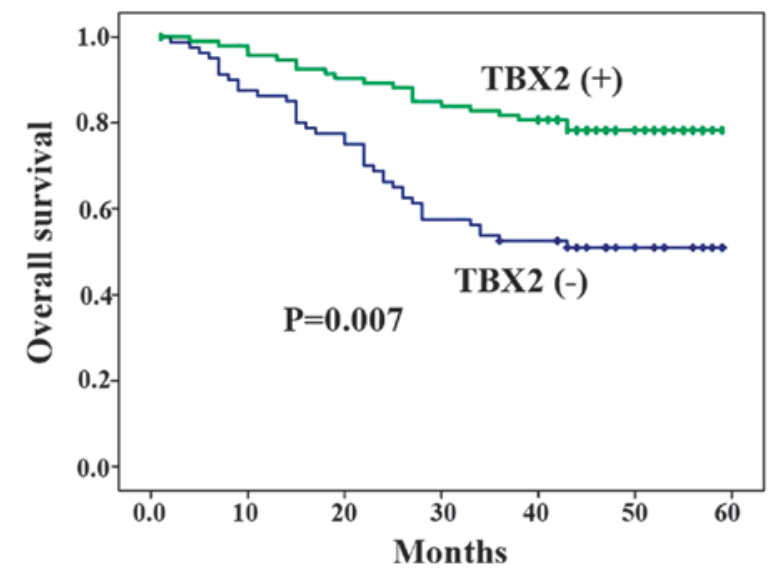

Figure 4. Kaplan-Meier survival curves of 266 patients with gastric cancer subsequent to gastrectomy. The survival of patients with TBX2 expression was significantly shorter compared with the survival of patients without TBX2 expression $(\mathrm{P}=0.007)$. TBX2, T-box 2 .

the suppression of senescence and control of invasiveness (11). TBX2, located at 17q23, has been revealed to be highly amplified and overexpressed in breast cancer cell lines through fluorescence in situ hybridization (FISH) (12). Jacobs et al (7) revealed that TBX2 was amplified in a subset of primary human breast cancers, indicating that this gene may contribute to breast cancer development. In addition, Vance et al (13) found that TBX2 was poorly expressed in the melanocyte cell line, while it was overexpressed in all melanoma cell lines tested. This study also found that TBX2 played an important role in maintaining cell proliferation and suppression of senescence in melanomas. Furthermore, Mahlamaki et al (9) detected that TBX2 was amplified in 50\% of pancreatic cancer cell lines tested by FISH. TBX2 was also overexpressed in several cancers, including breast and pancreatic cancers and melanoma, where TBX2 was demonstrated to function as a tumor-associated gene. Furthermore, Duo et al (14) reported previously in pancreatic cancer that TBX2 was closely associated with the degree of tumor differentiation, increased TNM stage and presence of distant metastasis. However, extremely little is known on the deregulation of TBX2 in GC.

In the present study, TBX2 expression was measured in fresh frozen specimens of GC and it was found that the expression levels of TBX2 mRNA and protein in GC tumor tissues were increased compared with the corresponding non-cancerous mucosa. It was suggested that TBX2 was upregulated at the transcriptional and post-transcriptional levels. Additional validation by immunohistochemistry revealed that $66.2 \%$ (176/266) of GC tissues exhibited positive staining, while only $8.27 \%(22 / 266)$ of the matched non-cancerous tissues were immunoreactive for TBX2. These data indicated that TBX2 may be involved in the progression of gastric carcinogenesis. In the present study, the association between TBX2 expression and the clinicopathological features of GC was also analyzed. The current results revealed a correlation between the expression of TBX2 in tumors and the clinical tumor stage, and a correlation between vascular invasion and distant metastasis. These data indicate that upregulated expression of TBX2 may contribute to the invasion and metastasis of tumors. Therefore, TBX2 may be a possible biomarker for the 
identification of subsets of colon cancer with a more aggressive phenotype. Consistent with the present results, Dimova et al (15) identified TBX2 to be a specific genomic marker for late-stage ovarian cancers. Similarly, Kandimalla et al (16) recognized TBX2 expression as a pTa stage-specific prognostic marker in bladder cancer.

Overall, the present study revealed that patients with tumors expressing TBX2 demonstrated a worse survival rate compared with patients without TBX2 expression. In addition, TBX2 expression was strongly associated with an increased risk of tumor invasion and metastasis compared with the absence of TBX2 expression. Using the univariate and multivariate Cox model analysis, it was confirmed that TBX2 may act as a significant independent prognostic factor for patients with GC.

\section{References}

1. Liang JW, Gao P, Wang ZN, Song YX, Xu YY, Wang MX, Dong YL and Xu HM: The integration of macroscopic tumor invasion of adjacent organs into TNM staging system for colorectal cancer. PLoS One 7: e52269, 2012.

2. Jemal A, Bray F, Center MM, Ferlay J, Ward E and Forman D: Global cancer statistics. CA Cancer J Clin 61: 69-90, 2011.

3. Sun J, Jiang T, Qiu Z, Cen G, Cao J, Huang K, Pu Y, Liang H, Huang R and Chen S: Short-term and medium-term clinical outcomes of laparoscopic-assisted and open surgery for colorectal cancer: A single center retrospective case-control study. BMC Gastroenterol 11: 85, 2011.

4. Popa F, Bratucu M and Radu P: Present and future tense in operable rectal cancer. Chirurgia (Bucur) 106: 11-16, 2011.

5. Rowley M, Grothey E and Couch FJ: The role of Tbx 2 and Tbx 3 in mammary development and tumorigenesis. J Mammary Gland Biol Neoplasia 9: 109-118, 2004.

6. Abrahams A, Parker MI and Prince S: The T-box transcription factor Tbx2: Its role in development and possible implication in cancer. IUBMB Life 62: 92-102, 2010.
7. Jacobs JJ, Keblusek P, Robanus-Maandag E, Kristel P, Lingbeek M, Nederlof PM, van Welsem T, van de Vijver MJ, Koh EY, Daley GQ and van Lohuizen M: Senescence bypass screen identifies TBX2, which represses Cdkn2a (p19(ARF)) and is amplified in a subset of human breast cancers. Nat Genet 26: 291-299, 2000.

8. Vance KW, Carreira S, Brosch G and Goding CR: Tbx2 is overexpressed and plays an important role in maintaining proliferation and suppression of senescence in melanomas. Cancer Res 65: 2260-2268, 2005.

9. Mahlamäki EH, Bärlund M, Tanner M, Gorunova L, Höglund M, Karhu R and Kallioniemi A: Frequent amplification of $8 \mathrm{q} 24,11 \mathrm{q}, 17 \mathrm{q}$ and $20 \mathrm{q}$-specific genes in pancreatic cancer. Genes Chromosomes Cancer 35: 353-358, 2002.

10. Salako SE: The declaration of Helsinki 2000: Ethical principles and the dignity of difference. Med Law 25: 341-354, 2006.

11. Wansleben S, Peres J, Hare S, Goding CR and Prince S: T-box transcription factors in cancer biology. Biochim Biophys Acta 1846: 380-391, 2014

12. Bärlund M, Monni O, Kononen J, Cornelison R, Torhorst J, Sauter G, Kallioniemi OLLI-P and Kallioniemi A: Multiple genes at $17 \mathrm{q} 23$ undergo amplification and overexpression in breast cancer. Cancer Res 60: 5340-5344, 2000.

13. Vance KW, Carreira S, Brosch G and Goding CR: Tbx2 is overexpressed and plays an important role in maintaining proliferation and suppression of senescence in melanomas. Cancer Res 65: 2260-2268, 2005.

14. Duo S, Tiao-Dong T, Lei Z, Wei W, Hong-Li S and Xian-Wei D: Expression and clinical significance of tbx 2 in pancreatic cancer. Asian Pac J Cancer Prev 10: 118-122, 2009.

15. Dimova I, Orsetti B, Negre V, Rouge C, Ursule L, Lasorsa L, Dimitrov R, Doganov N, Toncheva D and Theillet C: Genomic markers for ovarian cancer at chromosomes 1,8 and 17 revealed by array CGH analysis. Tumori 95: 357-366, 2009.

16. Kandimalla R, van Tilborg AA, Kompier LC, Stumpel DJ, Stam RW, Bangma CH and Zwarthoff EC: Genome-wide analysis of $\mathrm{CpG}$ island methylation in bladder cancer identified TBX2, TBX3, GATA2 and ZIC4 as pTa-specific prognostic markers. Eur Urol 61: 1245-1256, 2012. 